\title{
COMPREHENSIVE ORGANOMETALLIC ANALYSIS
}




\section{COMPREHENSIVE ORGANOMETALLIC ANALYSIS}

T. R. Crompton

North West Water Authority

Warrington, England 
Library of Congress Cataloging in Publication Data

Crompton, T. R. (Thomas Roy)

Comprehensive organometallic analysis.

Bibliography: $\mathbf{p}$.

Includes index.

1. Organometallic compounds-Analysis. I. Title.

QD411.C7625 1987

$547^{\prime} .05046$

ISBN-13: 978-1-4615-9500-7 e-ISBN-13: 978-1-4615-9498-7

DOI: $10.1007 / 978-1-4615-9498-7$

(C) 1987 Plenum Press, New York

Softcover reprint of the hardcover 1st edition 1987

A Division of Plenum Publishing Corporation

233 Spring Street, New York, N.Y. 10013

\section{All rights reserved}

No part of this book may be reproduced, stored in a retrieval system, or transmitted in any form or by any means, electronic, mechanical, photocopying, microfilming, recording, or otherwise, without written permission from the Publisher 
To

Elisabeth

on our

Silver Wedding Anniversary 


\section{PREFACE}

It is now some sixteen years since the author's first series of books on the analysis of organometallic compounds. Many developments in the subject have occurred since that time and a new book on the subject is now overdue.

The present book aims to provide a comprehensive review of the subject. It covers not only all aspects of the analysis of organometallic compounds but also contains two additional chapters, dealing with environmental analysis and the use of chelates of metals in the determination of very low concentrations of organic metals.

Whilst reviewing the literature for the present book, it was observed that whereas papers published prior to 1973 dealt almost exclusively with various forms of analysis, a high proportion of those published during the past ten years were concerned with the application of proven or newly developed methods to the determination of organometallic compounds in environmental samples such as water, air, soil, river and ocean sediments, fish life and biota samples. An increasing range of elements including mercury, lead, arsenic, tin, antimony, selenium and manganese are now being found in organically bound forms in the environment, some resulting from pollution, others formed in nature by bacterial processes. As many of these substances have appreciable implications to human and animal health and the ecosystem as a whole, it was considered that it would be timely to include a separate chapter in the book devoted entirely to this subject.

Many elements, upon reaction with particular chelating agents, produce chelates which are amenable to chromatographic analysis and, in many cases, are sufficiently volatile to be gas chromatographed. This has opened up a whole new area of analysis of mixtures of metals at very low concentrations.

In many instances chelate formation-gas chromatographic methods have absolute detection limits several orders of magnitude lower than those achieved by competing techniques such as atomic absorption, neutron activation analysis, emission spectrography and spark-source mass spectrometry.

The purpose of this separate chapter is to gather together the world literature on this new subject so as to enable analytical chemists to take 
a strong interest in and develop further, the technique which, in the opinion of the author, has even greater potential in the future.

The first seven chapters of the book cover each of the major analytical techniques that can be applied to the analysis of organometallic compounds. Within each chapter, the elements are discussed in alphabetical order. The first two chapters cover the determination of elements and functional groups. Suceeding chapters, respectively, cover the applications of titration techniques, visible and ultra-violet spectroscopic techniques, other spectroscopic techniques particularly infrared, NMR, PMR, etc., polarography, gas chromatography and finally a chapter covering other chromatographic techniques.

Errors are inevitable in a work of this size; the author would be grateful to receive notification of any errors so that they can be rectified in future editions.

It is hoped that this book will serve its aim of being a source-book of all aspects of the analysis of organometallic compounds, their occurrence in the environment and their uses in analytical chemistry.

The volumes will interest workers in a wide variety of fields both industrial and academic, at both the pure and the applied ends of the subject. In addition to analytical chemists the book will be of interest to organic chemists and those concerned with the environment and public health. Organometallic chemistry is a field which continues to grow and to which students should direct their interest and it is hoped that they will find much to interest them.

T. R. Crompton 
ACKNOWLEDGEMENTS

The author wishes to express his gratitude to the publishers of various journals for permission to reproduce the following illustrations.

\section{FIGURE}

1,2,5,6,7 T.R. Crompton, Chemical Analysis of Organometallic Compounds, Vol. V, Academic Press, London, New York, San Francisco, 1977, Chapter 20, pp. 115-235.

3 R. Dijkstra and E.A.M. Dahmen, Z. Ana1. Chem。, 181, 399 (1961).

4 B.J. Phillip, W.L. Mundry, and S.C. Watson, Ana1. Chem., 45, 2298 (1973).

8 I. Dunstan and J.V. Griffiths, Anal. Chem. 33, 1598 (1961)。

9 H. Allen and S. Tannenbaum, Anal. Chem., 31, 265 (1959).

10 S.A. Greene and H. Pust, Anal. Chem., 30, 1039 (1958).

11 J.C. Boaker and T.L. Isenhour, Ana1. Chem., 41, 1705 (1969).

12 H. Pieters and W.J. Buis, Mickrochem。 J., 8, 383 (1964).

13,14 R.A. Mostyn and A.E. Cunningham, J. Inst. Pet., 53, 101 (1967).

15 B.C. Southworth, J.H. Hodecker, and K.D. Fleischer, Anal. Chem., 30, 1152 (1958).

16 I. Lysyj and J.E. Zarembo, Microchem. J., 2, 245 (1958).

17,18 B.D. Holt, Anal. Chem., 37, 751 (1965).

19 O. Meier and N. Shaltiel, Mikrochim Acta 580 (1960).

20 K. Ziegler H. Gellert and Justus Liebigs, Ann. Chem., 629, 20 (1960).

21 T.R. Crompton, Analyst (London), 91, 374 (1966).

22 D.F. Hagen and W.D. Leslie, Anal. Chem., 35, 814 (1963).

23 E. Bonitz, Chem. Ber., 88, 742 (1955).

24 M. Farina, M. Donati and H. Ragzzini, Ann. Chim., (Rome), 48. 501 (1958).

25 L. Nebbia and B. Pagani, Chim. Ind. (Milan), 44, 383 (1962).

26 E.H. Hoffman and W. Tornau, Z. Anal. Chem. 186, 231 (1962).

27,28 M. Dimbat and G.A. Harlow, Anal. Chem., 34, 450 (1962).

29,30,34 W.L. Everson, Anal. Chem., 36, 854 (1964).

31 E.G. Hoffman and W. Tornau, Z., Ana1. Chem., 188, 321 (1962).

32 G. Pilloni and G. Plazzogna, Ana1. Chim. Acta, 35, 325 (1966).

33 A.F. Clifford and R.R. Olsen, Ana1. Chem., 32, 544 (1960).

35 S.C. Watson and J.F. Eastham, Ana1. Chem., 39, 171 (1967)。 
36 M. Dimbat and G.R. Harlow, Anal. Chem., 34, 450 (1962)。

37 C. Jolibois, R. Acad. Sci., 155, 213 (1912).

38,39 G. Tagliavini, Anal. Chim. Acta, 34, 24 (1966).

40,41 W.L. Everson and E.M. Ramirez, Anal. Chem., 37, 812 (1965).

42,43 E. Bonitz, Chem. Ber., 88, 742 (1955).

44 W.P. Neumann, Angew. Chem., 69, 730 (1957).

45 J.H. Mitchen, Anal. Chem., 33, 1331 (1961).

46,47,48 B.T. Commins and P.J. Lawther, Br. J. Ind. Med., 22, 139 (1965).

49 J. Stary, K. Kratzer and K.J. Prasilova, Anal. Chim. Acta, 100,627 (1978).

50 S. Sass, W.D. Ludemann, B. Witten, V. Fishen, A.J. Sisti, and J.I. Miller, Anal。Chem., 29, 1346 (1957)。

51 K.S. Pitzer and R.J. Sheline, J. Chem. Phys., 16, 552 (1948).

52,53,61 E.G. Hoffman, Z. E1ektrochem., 64, 616 (1960).

54,155 E.G. Hoffman and G. Schomerg, Z. fur Elecktrochemie 61, 1101 (1957).

56 E.G. Hoffman and G. Schomberg, Z. fur Elecktrochemie, 61, 1110 (1957).

57 J.V. Bell, J. Heisler, H. Tannenbaum, and J. Goldenson, Anal. Chem., $\underline{25}, 1720$ (1953).

58 D.V. Guertin, S.E. Wiberley, W.H. Bauer, and J. Golderson, J. Phys. Chem., 60, 1018 (1956)。

59 V. Yamamoto, Bull. Chem. Soc. Japan, 35, 619 (1962).

60 J. Smidt, M.P. Gruenewage, and H. de Vries, Rec. Irav. Chim, 81,729 (1962).

62 J.J. Kaufman, W.S. Koski, L.T. Kuhns, and S.S. Wright, J. Amer. Chem. Soc., 85, 1369 (1963).

63,64 W.S. Koski, J.J. Kaufman, and P.C. Lanterbur, J. Amer. Chem. Soc., 79,2382 (1957).

65 A.F. Reid, D.E. Scaife, and P.C. Wailes, Spectrochim. Acta, 20, 1257 (1964).

66 J.R. Urwin and P.J. Reed, J. Organometal Chem., 15, 1 (1968).

67,68 H.Susi and H.E. Rector, Anal. Chem., 30, 1933 (1958).

69 J.H. Lowry, R.B. Smart, and K.H. Mancy, Anal. Chim•, 50, 1303 (1978).

70 J.E. de Vries, A. Lauw-Zecha, and A. Pellecer, Anal. Chem., 31, 1995, (1959).

71 M. Mehner, H. Jehring, and H. Kriegsmann, 3rd Analytical Conference, Budapest, 24-29 August, 1970.

72,73 R. Geyer and H.T. Seidlitz, Z. Chem., 4, 468 (1964)。

74 L.M. Brown and K.S. Mazdiynasi, Anal. Chem., 41, 1243 (1969).

75, 76, 79 B.J. Gudzinowicz and J.L. Driscol1, J. Gas Chromatogn, 1, 25 (1963)。

77,78 G.E. Parris, W.R. Blair, and J.E. Brinkman, Anal. Chem., 49, 2215 (1977).

80,81,82 C. Feldman and D.A. Batistoni, Anal. Chem. 49, 2215. (1977).

83 G. Schomberg, R. Koster, and D. Henneberg, Z. Anal. Chem., 170, 285 (1959). 
84 G.R. Seely, J.P. Oliver, and D.M. Ritter, Anal. Chem., 31, 1993 (1959).

85 T.D. Parsons, M.B. Silverman, and D.M. Ritter, J. Am. Chem. Soc., 79, 5091 (1957).

86 J.A. Semlyen and C.S.G. Phillips, J. Chromatography, 18, 1 (1965).

87,88 J.J. Kaufman, J.E. Todd, and W.S. Koski, Anal. Chem., 29, 1032 (1957).

89,90 H.W. Myers and R.F. Putman, Anal. Chem., 34, 664 (1962).

91,92 H. Veening, J. Graver, D.B. Clark, and B.R. Willeford, Anal. Chem., 41, 1655 (1969).

93 H. Veening, J.S. Keller, and B.R. Willeford, Anal. Chem., 43, 1516 (1971)。

94 W.J.A. Van der Heuvel, J.S. Keller, H. Veening, and B.R. Willeford, Analyt. Lett., 3 , 279 (1970).

95 C.S.G. Phillips and P.L. Timms, Anal. Chem., 35, 505 (1963).

96 B. Iatridis and G. Parissakis, Anal. Chem., 49, 909 (1977).

97 O.E. Ayers, T.C. Smith, J.D. Burnett, and B.W. Pouder, Anal. Chem. 38, 1606 (1966).

98,99,100 J.E. Lovelock and A. Zlatk1s, Anal. Chem. 33, 1958 (1961).

101,102 HoJ. Dawson, Anal. Chem., 35, 542 (1963).

103 E. Barrall and P. Ballinger, J. Gas Chromatography, 1, 7 (1963).

104 E. Bonnelli and H. Hartmann, Anal. Chem., 35, 1980 (1963).

105,106 N.L. Soulages, Anal. Chem., 38, 28 (1966).

107 W.S. Leonhardt, R.C. Morrison, and W.C. Kamicnski, Anal. Chem., 38, 466 (1966).

108 L.V. Giold, C.A. Hollingsworth, D.H. McDaniel, and J.H. Wotiz, Anal. Chem., 33, 1156 (1961).

109 A. Wowk and S. Di. Giovanni, Anal. Chem., 38, 742 (1966).

110,111 P.C. Uden, R.M. Barnes, and P. Di. Sanzo, Anal. Chem., 50, 852, (1978).

112,113 B.D. Quimby and P.C. Uden, Anal. Chem., 50, 2112 (1978).

114 A. Apelblat and A. Hornik, J. Chromatography, 24, 175 (1966)。

115 A. Apelblat and A. Hornik, Trans Faraday Soc. No., 529, 63, 185 (1967).

116 A. Apelblat, J. Inorg. Nucl。Chem., 31, 483 (1969).

117 R.L. Grob and G.L. McCrea, Anal. Lett., 1, 55 (1967).

118 K.P. Berlin, T.H. Austin, M.E. Nagahushanam, J. Peterson, J. Calvert, W.A. Wilson, and D. Hopper, J. Gas Chromatogr., 3, 256 (1965).

119 A. Davis, A. Roadi, J.G. Michalovic, and A.M. Joseph, J. Gas Chromatography, 1,23 (1963).

120 C.S. Evans and C.M. Johnson, J. Chromatography, 21, 202 (1966).

121,122 C.S.G. Phillips, P.L. Timms, Anal。Chem., 35, 505 (1963).

123 F.H. Pollard, G. Nickless, and P.C.Uden, J. Chromatogr., 19, 28 (1965).

124 H. Rotzsche, Z. Anorg. Chem., 324, 197 (1963). 
125 H. Rotzsche, Z. fur Anorg. und Allgemeine. Chemie., 328, 79 (1964).

126 M. Wurst, Col1. Czech. Chem. Commun., 30, 2038 (1965).

127 J.B. Carmichael, D.J. Gordon, and C.E. Ferguson, J. Gas Chromatography, 4 , 347 (1966).

$128,129,130$ M.1. Osman, H.H. Hill, M.W. Holdren, and H.H. Wetberg, Anal. Chem., 51, 1286 (1979).

$131,132,133$ G. Garzo and F. Till, Talanta, 10, 583 (1963).

134 D. Thrash L. Viosinet and K.E. Williams, J. Gas Chromatography July 248 (1965).

135,136 F.H. Pollard, G. Nickless, and D.B. Thomas, J. Chromatogr., 22, 286 (1966).

137 D.D. Schlenter and S. Siggia, Anal. Chem, 49, 2343 (1977).

138 D.D. Schleuter, Ph.D. Dissertation, University of Massachusetts, Amherst, Massachusetts (1976).

139,140 F.H. Pollard, G. Nicklett, and D.J. Cooke, J. Chromatography, 13, 48 (1964).

141 R.C. Putnam and H. Put, J. Gas Chromatography, $\underline{3}, 2$ (1965) and $\underline{3}, 2$ (1965).

142 H. Geissler and H. Kriegsmann, Z. Chemfe, Lpz., 4, 354 (1964).

143, 144 B.L. Tonge, J. Chromatography, 19, 182 (1965).

145,146,147 S.G. Perry, J. Gas Chromatography, 93 March (1964).

148 J.E. Schwarberg, R.W. Moshier, and J.H. Walsh, Talanta, 11, 1213 (1964).

149 J.E. Schwarberg, Master's thesis, University of Dayton, 1964.

150 P. Jacquelot and G. Thomas, Bull Soc. Chim. Fr. 702 (1971).

151 H. Veening and J.F.K. Huber, J. Gas Chromatog., 6, 326 (1968).

152,153 J.A. Stokeley, Master's thesis, Oak Ridge University, Diss. Abstr., 27, 1388 B (1966).

154 K.J. Eisentraut and R.E. Sievers, J. Am. Chem. Soc., 87, 5254 (1965).

155 Anonymous, Chem. Eng. News, 43, November 22, 39 (1965).

156 T. Shigematsu, M. Matsui, and K. Utsunomiya, Bu11. Inst. Chem. Res., Kyoto Univ., 46, 256 (1968).

157 W.I. Stephen, I.J. Thompson and P.C. Uden, Chem. Commun., pp. 269-270 (1969).

158 A. Khalique, W.I. Stephen, P.E. Henderson, and P.C. Uden, Anal. Chim., Acta, 101, 117 (1978).

159 T. Fujinaga and Y. Ogino, Bull. Chem. Soc., Japan, 40, 434 (1967).

160 J.K. Foreman, T.A. Gough, and E.A. Walker, Analyst (London), 95, 797 (1970).

161 R. Belcher, C.R. Jenkins, W.I. Stephen, and P.C. Uden, Talanta, 17, 455 (1970).

162,163 T.J. Cardwe11, D.J. Resarro, and P.C. Uden, Anal. Chim. Acta, 85, 415 (1976).

164 Y. Shimoishi and K. Toei, Anal. Chim. Acta, 100, 65 (1978). 
165 E.G. Gaetani, C.F. Laureri, A. Magnia, and G. Parolari, Anal. Chem., 48, 1725 (1976).

166,167 T.J. Cardwe11, D. Caridi, and M.S. Loa, J. Chromatography, 351, 331 (1986).

168 M. Saitoh, R. Kurada, and M. Shibukawa, Anal. Chem., 55, 1025 (1983).

169 K. Saitoh, M. Kabayashi, and N. Suzuki, Anal. Chem. 53, 2309 (1981).

170 G.R. Ricci, L.S. Shepard, G. Colovos, and N.H. Hester, Anal. Chem., 53, 610 (1981).

171 J.D. Messman and T.C. Rains, Anal. Chem., 53, 1632 (1981).

172 G. Misson, Chem. Ztg., 32, 633 (1908).

173 R.B. Lew and F. Jakob, Talanta, 10, 322 (1963).

174 Department of the Environment and National Water Council (U.K.) H.M. Stationary Office, London, 23 pp (pt 23 Abenv) (1978).

175 K. Minagawa, Y. Takizawa, and I. Kufune, Anal. Chim. Acta., 103, 115 (1980).

176,177 K. Chiba, K. Yoshida, K. Tanabe, H. Horaguchi, and K. Fuwa, Ana1. Chem., 55, 450 (1983).

178 G.A. Hambrick, P.N. Froebich, O.A. Meirate, and B.L. Lewis, Anal. Chem., 56, 421 (1984).

179 Y.K. Chau, P.T.S. Wong, and G.A. Bengert, Anal. Chem., 54, 246 (1982).

180 C.J. Soderquist and D.G. Crosby, Anal. Chem. 50, 1435 (1978).

$181,182,183$ R.S. Raman and M.A. Tomkins, Anal. Chem., 51, 12 (1979).

184 Y.K. Chau, P.T.B. Wong, and O. Kramar, Anal. Chem. Acta., 146, 211 (1983).

185,186 M.0. Andreae, Anal. Chem., 49, 820 (1977).

187 A.A. Grabinski, Anal. Chem., 53, 966 (1981).

188,189 J. Aggett, R. Kadwani, Analyst (London), 108, 1495 (1983).

190 C.E. Stringer and M. Attrep, Anal. Chem., 51, 731 (1979).

191,192 L. Brown, S.J. Haswell, H.M. Rhead, P.O'Neill, and C.C. Bancroft, Analyst, 108, 1511 (1983).

193,194,195 Y.K. Chau, P.T.S. Wong, and H. Saitoh, J. Chromatogr. Sci., 14,162 (1976).

196,197,198 S. Hanamura, B.W. Smith, and J.D. Winefordner, Anal. Chem., 55, 2026 (1983).

199,200,201 J.G. Gonzales, and R.T. Ross, Anal. Lett., 5, 683 (1972).

202 W.A. MacCrehan, R.A. Durst, and J.M.Bellama, Anal. Lett., 10, 1175 (1977).

203,204 C.J. Cappon and V. Crispin Smith, J. Anal. Chem., 49, 365 (1977).

205 M. Morita, T. Uehiro, K. Fuwa, Anal. Chem., 53, 1806 (1981).

206 M. Loe, R. Cruz, and J.C. Van Loon, Anal. Chim. Acta, 120, 171 (1980).

207 G. Torsi, F. Palmisano, Analyst (London), 108, 1318 (1983).

208 G. Torsi, E. Desimoni, F. Palmisano, Analyst (London), 107, 96 (1982).

209 J.W. Robinson, E.L. Kicsel, J.P. Goodbread, R. Bliss, and R. Marsha11, Anal. Chim. Acta, 92, 321 (1977). 
210,211 A.J. McCormack, S.C. Tong, and W.D. Cooke, Anal. Chem., 37, 1470 (1965).

212 W.R.A. De. Jonghe, D. Chakraborti, and F.C. Adams, Anal. Chem., $\underline{52}$, 1974 (1980).

213 H. Koizumi, R.D. McLaughlin, and T. Hadeishi, Anal. Chem., 51, 387 (1979).

214,215 R。 Moss and E.V. Browett, Analyst (London), 91, 428 (1966). 


\section{CONTENTS}

CHAPTER 1 DETERMINATION OF ELEMENTS AND FUNCTIONAL GROUPS

Organoaluminium Compounds . . . . . . . . . . . . . . . . 1

A. Determination of Aluminium (1)

B. Determination of Carbon and Hydrogen (3)

C. Determination of Aluminium-bound Halogens (4)

D. Determination of Aluminium-bound Alkyl Groups up to Butyl and Hydride Groups (5)

E. Determination of Higher Aluminium-bound Alkyl Groups (21)

F. Determination of Higher Alkyl and Alkoxide Groups (22)

G. Determination of Aluminium-bound Alkoxide Groups up to Butoxy (23)

H. Determination of Aluminium-bound Amino Groups (26)

I. Determination of Aluminium-bound Thioalkoxide Groups (26)

Organoantimony Compounds . . . . . . . . . . . . . . . . .

A. Determination of Antimony (27)

Organoarsenic Compounds . . . . . . . . . . . . . . . . . . . .

A. Determination of Arsenic (28)

B. Determination of Carbon (33)

C. Determination of Fluorine (33)

D. Determination of Sulphur (33)

Organoberyllium Compounds . . . . . . . . . . . . . . .

A. Determination of Carbon and Hydrogen (33)

Organobismuth Compounds . . . . . . . . . . . . . . . .

A. Determination of Bismuth (34)

Organoboron Compounds . . . . . . . . . . . . . . . . . .

A. Determination of Boron, Carbon and Hydrogen (34)

B. Determination of Chlorine (44)

C. Determination of Nitrogen and Boron (46)

D. Determination of Hydride and Active Hydrogen (48)

Organocalcium Compounds .................... . 48

A. Determination of Carbon and Hydrogen (48)

Organochromium Compounds. . . . . . . . . . . . . . . .

A. Determination of Chromium (50)

Organocobalt Compounds . . . . . . . . . . . . . . . .

A. Determination of Cobalt (51)

Organocopper Compounds .....................

A. Determination of Copper (53) 
Organogermanium Compounds . . . . . . . . . . . . . 53

A. Determination of Germanium (53)

B. Determination of Carbon and Hydrogen (54)

C. Determination of Alkoxy Germanes and Mercaptogermanes (54)

Organoiron Compounds .....................

A. Determination of Iron (58)

Organolead Compounds . . . . . . . . . . . . . . . . .

A. Determination of Lead (59)

B. Determination of Organolead Compounds in Petroleum via the determination of Lead (59)

C. Determination of Carbon and Hydrogen (69)

D. Determination of Halogen (69)

Organolithium Compounds

A. Determination of Lithium, Carbon, Hydrogen and Oxygen (69)

Organomagnesium Compounds

A. Determination of Carbon and Hydrogen (70)

B. Determination of A1kyl Groups (70)

Organomanganese Compounds

A. Determination of Manganese (70)

Organomercury Compounds

A. Determination of Mercury (70)

B. Determination of Carbon, Sulphur, Halogens and Oxygen (75)

C. Determination of Carbon, Hydrogen and Mercury (78)

D. Determination of Oxygen (81)

Organonickel Compounds . . . . . . . . . . . . . . . . .

A. Determination of Nickel (82)

Organopalladium Compounds

A. Determination of Palladium and Chloride (82)

Organophosphorus Compounds . . . . . . . . . . . . . . .

A. Determination of Phosphorus (83)

B. Determination of Iron, Silicon, Titanium, Arsenic and Phosphorus (98)

C. Determination of Carbon and Hydrogen (98)

D. Determination of Nitrogen (101)

E. Determination of Oxygen (101)

F. Determination of Halogens (102)

G. Determination of Sulphur (108)

Organoplatinum Compounds .................. 108

Organopotassium Compounds . . . . . . . . . . . . . 108

Organoselenium Compounds . . . . . . . . . . . . . . 109

A. Determination of Selenium (109)

B. Determination of Carbon and Hydrogen (111)

Organosilicon Compounds . . . . . . . . . . . . . . 111 Determination of Elements (111)

A. Determination of Silicon (111)

B. Determination of Phosphorus and Silicon (119) 
C. Determination of Aluminium and Silicon . . . . . . . . 122

D. Determination of Titanium, Silicon, Phosphorus and Aluminium (124)

E. Determination of Tin, Silicon and Titanium (125)

F. Determination of Hydrogen, Silicon and Halogen (125)

G. Determination of Halogens (125)

Determination of Functional Groups (127)

H. Determination of Alkoxy and Aryloxy Groups (131)

I. Determination of Acetoxy Groups (133)

J. Determination of Silicon bound Hydroxy Groups (134)

K. Determination of Mercaptosilanes (137)

L. Determination of Silidyne Groups (138)

M. Determination of Unsaturation (140)

N. Determination of Phenyl and Alkyl Groups (140)

0 . Determination of Hydrocarbon Constituents (141)

P. Non-Aqueous Titrimetry (141)

Organothallium Compounds . . . . . . . . . . . . . . . . 142

A. Determination of Carbon, Hydrogen and Thallium (142)

Organotin Compounds . . . . . . . . . . . . . . . . 143

A. Determination of Tin (143)

B. Determination of Carbon and Hydrogen (144)

C. Determination of Halogens (145)

D. Determination of Nitrogen (145)

E. Determination of Sulphur (145)

Organotitanium Compounds . . . . . . . . . . . . . . . 145

A. Determination of Titanium, Iron, Phosphorus, Silicon and Chlorine (145)

Organozinc Compounds . . . . . . . . . . . . . . . . . . 146

A. Determination of Zinc (146)

B. Determination of Halogens (146)

C. Determination of Zinc-bound Alkoxy Groups (147)

D. Determination of Lower Alkyl and Hydride Groups (147)

Organozirconium Compounds . . . . . . . . . . . . . . . 148

CHAPTER 2 TITRATION PROCEDURES

Organoaluminium Compounds .. . . . . . . . . . . . . 149

A. Classical Titration Procedures (149)

B. Conductiometric Titration (157)

C. Potentiometric Titration (160)

D. Amperometric Titration (164)

E. Activity by Dielectric Constant Titration (164)

F. Lumometric Titration (165)

G. Thermometric Titration (167)

Organoarsenic Compounds . . . . . . . . . . . . . . 172

Organoboron Compounds . . . . . . . . . . . . . . . 173

A. Classical Titration Procedures (173)

B. Potentiometric Titration (174)

Organocalcium Compounds . . . . . . . . . . . . . . 174

Organocobalt Compounds . . . . . . . . . . . . . . . 174 
Organocopper Compounds . . . . . . . . . . . . . . . 174

Organogermanium Compounds . . . . . . . ....... 175

Organoiron Compounds . . . . . . . . . . . . . . . 175

A. Classical Titration Procedures (175)

B. Potentiometric Titration (175)

Organolead Compounds

A. Classical Titration Procedures (176)

B. Amperometric Titration (180)

C. Conductiometric Titration (180)

D. Coulometric Titration (180)

E. High frequency Titration (181)

Organolithium Compounds .................. 181

A. Classical Titration Procedures (181)

B. Potentiometric Titration (188)

C. Thermometric Titration (189)

D. High frequency Titration (191)

E. Lumometric Titration (193)

Organomagnesium Compounds ................. 193

A. Classical Titration Procedures (193)

B. Amperometric Titration (195)

C. Potentiometric Titration (195)

Organomercury Compounds

A. Classical Titration Procedures (196)

B. Potentiometric Titration (197)

C. Coulometric Titration (198)

Organophosphorus Compounds . . . . . . . . . . . . 198

A. Esters of Phosphoric Acid (198)

B. Esters of Phosphorous Acid (199)

C. Esters of Phosphonic, Phosphinic and Phosphorous Acids (200)

D. Esters of Pyrophosphoric Acid, Pyrophosphonic Acid, Phosphoroanhydrides, Alkyl-phosphonochloridates, Dialkylphosphoroch loridates and Alkylphosphonofluoridates (200)

E. Organophosphorus Fluoridates and Chloridates (204)

F. Phosphonium Salts and Phosphoranes (206)

G. Substituted Phosphines (208)

H. Trisubstituted Phosphine Oxides and Sulphides and bis (disubstituted phosphinyl) Alkanes (209)

I. Dialkylphosphorodithioates, Dialkyldithiophosphates, Phosphorothioates, Dialkylphosphonodithioates and other P - S Compounds (209)

J. Alkyl Phosphonic Chlorides

K. Alkyl Silylphosphates

Organopotassium Compounds

Organoselenium Compounds .................. 212

Organosodium Compounds ................... 212

Organosilicon Compounds . . . . . . . . . . . . . 212

A. Non-aqueous Titration (212)

B. Non-aqueous Conductiometric Titration (213)

C. Non-aqueous Amperometric Titration (213) 
Organotin Compounds . . . . . . . . . . . . . . . . 215

A. Classical Titration Procedures (215)

B. Potentiometric Titration (217)

C. Amperometric and Coulometric Titration (217)

Organozinc Compounds . . . . . . . . . . . . . . . . . 221

A. Classical Titration Procedures (221)

B. Thermometric Titration (222)

CHAPTER 3 SPECTROSCOPIC TECHNIQUES, VISIBLE AND ULTRAVIOLET SPECTROSCOPY

A. Visible Spectroscopy. . . . . . . . . . . . . . . 226

Organoaluminium Compounds (226)

Organoarsenic Compounds (232)

Organoboron Compounds (233)

Organochromium Compounds (234)

Organocobalt Compounds (235)

Organocopper Compounds (238)

Organogermanium Compounds (238)

Organoiron Compounds (238)

Organolead Compounds (245)

Mono-di-and Trisubstituted Lead Compounds (245)

Tetrasubstituted Lead Compounds (248)

Organomagnesium Compounds (249)

Organomanganese Compounds (253)

Organomercury Compounds (253)

Organonickel Compounds (261)

Organophosphorus Compounds (262)

Trialky1 phosphates (262)

Dialkyl Hydrogen Phosphites, Trialkyl and Triaryl Phosphites (262)

0,0-Dialky1-1-Hydroxyphosphonates (264)

Tetraethyl Pyrophosphate (265)

Phosphorophenoxides (265)

Phosphonium Salts (265)

Substituted Phosphine Oxides (266)

Alkyl Chloridates and Fluoridates and Alkylphosphoroamidocyanidate $(268)$

Dialkyldithiophosphoric Acids (272)

Glycerophosphates (273)

Organoselenium Compounds (274)

Organosilicon Compounds (274)

Organothallium Compounds (274)

Organotin Compounds (274)

Organozinc Compounds (280)

B. Ultraviolet Spectroscopy . . . . . . . . . . . . . . . 281

Urganoboron Compounds (281)

Organolithium Compounds (281)

Organomagnesium Compounds (281)

Organomercury Compounds (282)

Organosilicon Compounds (282)

Organotin Compounds (282)

CHAPTER 4 OTHER SPECTROSCOPIC TECHNIQUES

Organoaluminium Compounds . . . . . . . . . . . . . 283
A. Infrared Spectroscopy (283)
B. Raman Spectroscopy (296)
C. PMR and NMR Spectroscopy (301)
D. Mass Spectrometry (307) 
Organoboron Compounds . . . . . . . . . . . . . . . . 308

A. Infrared Spectroscopy (308)

B. NMR Spectroscopy (311)

C. Mass Spectrometry (312)

Organochromium Compounds

Mass Spectrometry (313)

Organocobalt Compounds .................. 313

Infrared Spectroscopy (313)

Organocopper Compounds

Mass Spectrometry (313)

Organogallium Compounds ................... 313

Electron probe Microanalysis (313)

Organogermanium Compounds . . . . . . . . . . . . . . . 314

A. Infrared Spectroscopy (314)

B. Mass Spectrometry (314)

Organoiron Compounds . . . . . . . . . . . . . . . . . 314

A. Infrared Spectroscopy (314)

B. NMR, PMR and ESR (317)

C. Mass Spectrometry (318)

D. X-Ray Diffraction and X-Ray Emission (318)

Organolead Compounds .................... 319

Infrared Spectroscopy (319)

Organolithium Compounds .................... 319 NMR Spectroscopy (319)

Organomanganese Compounds . . . . . . . . . . . . . 320

Infrared Spectroscopy (320)

Organomercury Compounds . . . . . . . . . . . . . . . 321

Infrared Spectroscopy (321)

Organonickel Compounds . . . . . . . . . . . . . . . . 321

Mass Spectrometry (321)

Organophosphorus Compounds ................. 321

A. Infrared Spectroscopy (321)

B. Mass Spectrometry (321)

C. NMR Spectroscopy (323)

Organosilicon Compounds ................... 323

A. Infrared Spectroscopy (323)

B. Specific Functional Groups (326)

C. Mass Spectrometry (338)

D. Raman Spectroscopy (338)

E. NMR Spectroscopy (339)

Organotin Compounds

A. Infrared and Raman Spectroscopy (339)

B. NMR Spectroscopy (341)

C. PMR Spectroscopy (342)

D. Mass Spectrometry (342) 
Organozinc Compounds . . . . . . . . . . . . . . . . . . 342 Infrared and Raman Spectroscopy (342)

\section{CHAPTER 5 POLAROGRAPHIC TECHNIQUES}

Organoarsenic Compounds . . . . . . . . . . . . . . . . . . . . . . . 344

Organoboron Compounds . . . . . . . . . . . . . . . . . . 346

Organochromium Compounds . . . . . . . . . . . . . . . 346

Organocobalt Compounds . . . . . . . . . . . . . . 346

Organoiron Compounds ................... . . 347

Organolead Compounds . . . . . . . . . . . . . . . . 347

Organomercury Compounds . . . . . . . . . . . . . . . . . . 349

Organonickel Compounds . . . . . . . . . . . . . . . . . . 351

Organophosphorus Compounds . . . . . . . . . . . . . . . . 351

Organoselenium Compounds . . . . . . . . . . . . . . 352

Organotin Compounds . . . . . . . . . . ...... . 352

Organozinc Compounds ................... . 357

\section{CHAPTER 6 GAS CHROMATOGRAPHY}

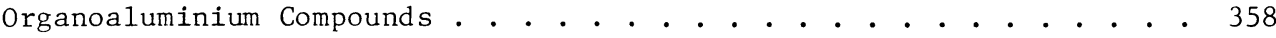

Organoantimony Compounds . . . . . . . . . . . . . . . . . 359

Organoarsenic Compounds . . . . . . . . . . . . . . . . . . . 359

A. Alkyl and Aryl Arsines (359)

B. Perfluoroorganoarsenic Compounds (361)

C. Detectors for Organoarsenic Compounds (364)

D. Miscellaneous Organoarsenic Compounds (368)

E. Arsine (368)

Organoberyllium Compounds . . . . . . . . . . . . . . . 370

Organoboron Compounds . . . . . . . . . . . . . . . 370

A. Boron Alkyls (370)

B. Borazdes (373)

C. Boron Hydrides (376)

D. Chloroboranes (379)

E. Boron Halides (380)

F. Methyl Borate (382)

G. Boronates (382)

H. Determination of B.H. Bonding (382)

Organochromium Compounds ................... . . 385

Organocobalt Compounds ................... 392

Organocopper Compounds . . . . . . . . . . . . . . 392

Organogallium Compounds . . . . . . . . . . . . . . . . . . 393

Organogermanium Compounds . . . . . . . . . . . . . . . . . . 393

A. Alkylgermanium Compounds (393)

B. Miscellaneous Organogermanium Compounds (395)

Organoiron Compounds . . . . . . . . . . . . . . . 396

A. Ferrocene and Derivatives (396)

B. Miscellaneous Organoiron Compounds (399)

Organolead Compounds . . . . . . . . . . . . . . . . 400

Organolithium Compounds ................... . 414

A. Viny1 Lithium (414)

B. Phenyl Lithium (415)

C. Methyl Lithium (415) 
Organomagnesium Compounds .................... 415

A. Alkyl and Aryl Magnesium Halides (415)

B. Vinyl Magnesium Halides (418)

C. Etio Phorphyrin Magnesium Chelates (418)

Organomanganese Compounds . . . . . . . . . . . . . . 418

Organomercury Compounds . . . . . . . . . . . . . . . 423

Organomolybdenum Compounds ................. . 425

Organophosphorus Compounds . . . . . . . . . . . . . . 426

A. Organophosphates and Thiophosphates (427)

B. Trialkyl and Triarylphosphites (433)

C. Trialkyl Phosphines, Phosphine Oxides and other oxygenated Organophosphorus Compounds (434)

D. Phosphoronitrilo Halides and Phosphorus Chlorides and Oxychlorides (438)

E. Insecticides and Pesticides (442)

Organoruthenium Compounds . . . . . . . . . . . . . 446

Organoselenium Compounds . . . . . . . . . . . . . . 446

Organosilicon Compounds . . . . . . . . . . . . . . 450

A. Silicon Hydrides (450)

B. Alkyl and Aryl Silanes (450)

C. Chlorosilanes (450)

D. Alkyl, Aryl and Vinyl Chlorosilanes (456)

E. Alkoxy Silanes (463)

F. Siloxanes (465)

G. Sily1 Ethers and Silyl Thioethers (469)

H. Miscellaneous Organosilicon Compounds (469)

I. Detectors for Organosilicon Compounds (472)

J. Micropyrolysis-Gas Chromatography (475)

Organotellurium Compounds . . . . . . . . . . . . . 4 486

Organotin Compounds . . . . . . . . . . . . . . . . . 487

A. Alkyltin and Vinyltin Compounds (487)

B. Organotin Halides (494)

C. Organotin Oxides and Hydroxides (498)

Organozinc Compounds ..................... 499

A. Alkylzinc Compounds (499)

B. Zincdialkyldithiophosphates (500)

CHAPTER 7 CHROMATOGRAPHY OF METAL CHELATES

A. Introductory (504)

B. Gas Chromatography, Acetyl Acetonates (506)

C. Gas Chromatography, Fluorinated Acetylacetonates (509)

D. Gas Chromatography, Hexafluoroacetylacetonates (516)

E. Gas Chromatography, $\beta$-Diketone Derivatives (518)

F. Gas Chromatography, Amino Substituted $\beta$-Diketonates (523)

G. Gas Chromatography, Dialkyldithiophosphates (527)

H. Gas Chromatography, Salicyaldimes (527)

I. Gas Chromatography, Diethyldithiocarbamates (527)

J. Gas Chromatography, Non-chelated Metal Complexes (527)

K. Gas Chromatography, Metal Porphyrins (528) 
L. Gas Chromotographic detection of Metal Chelates (530)

M. Gas Chromatography, Conclusions (537)

N. Liquid Chromatography of Metal Chelates (538)

0. Size Exclusion Chromatography of Metal Chelates (541)

P. High performance Thin-Layer Chromatography of Metal Chelates (542)

Q. Index of Metal Analysis by Gas Chromatography of their volatile Chelates (542)

Illustrative examples of the application of Chromatography to Metal Chelates (552)

R. Gas Chromatography (552)

S. High performance Liquid Chromatography (567)

T. Size-exclusion Chromatography (571)

U. High performance Thin-Layer Chromatography (578)

\section{CHAPTER 8 OTHER CHROMATOGRAPHIC TECHNIQUES}

A. Thin-layer Chromatography ................ 579

Organoantimony Compounds (579)

Organoarsenic Compounds (579)

Organobismuth Compounds (579)

Organoboron Compounds (579)

Organocobalt Compounds (580)

Organocopper Compounds (581)

Organoiron Compounds (581)

Organolead Compounds (582)

Organphosphorus Compounds (582)

Alkyl and Aryl Phosphates (582)

Alkyl and Aryl Phosphites (584)

Phosphonic Acids (584)

Phosphinic Acids (585)

Substituted Phosphines (585)

Substituted Phosphine Oxides (585)

Phosphorothioc Acid Esters (585)

Miscellaneous Organophosphorus Compounds (587)

Organophosphorus Insecticides and Pesticides (587)

Organosilicon Compounds (588)

Organotellurium Compounds (589)

Organotin Compounds (589)

Organozinc Compounds (592)

B. Paper Chromatography . . . . . . . . . . . . . . . 593

Organoarsenic Compounds (593)

Organobismuth Compounds (593)

Organocobalt Compounds (593)

Organocopper Compounds (594)

Organoiron Compounds (594)

Organolead Compounds (594)

Organomercury Compounds (595)

Organophosphorus Compounds (597)

Alkyl and Aryl Phosphates and Phosphites (597)

Phosphorous, Phosphinic and Phosphonic Acid Esters (601)

Trialkyl, Triaryl Phosphine and Phosphine Oxides (602)

Pyrophosphoric Acid Esters (602)

Phenylphosphorothioates (603)

Carbohydrate Esters of Phosphoric Acid (603)

Miscellaneous Organophosphosphorus Compounds (604)

Organophosphorus Insecticides and Pesticides (605) 
Organoselenium Compounds (605)

Organosilicon Compounds (606)

Organotin Compounds (611)

C. Column Chromatography . . . . . . . . . . . . . 613

Organoarsenic Compounds (613)

Organocobalt Compounds (614)

Organoiron Compounds (615)

Organolead Compounds (616)

Organomercury Compounds (619)

Organonickel Compounds (619)

Organophosphorus Compounds (620)

Phosphoric Acid Esters (620)

Alkyl Chloridates and Fluoridates (620)

Carbohydrate Phosphates (624)

Miscellaneous Organophosphorus Compounds (624)

Organophosphorus Insecticides and Pesticides (624)

Organoselenium Compounds (625)

Organosilicon Compounds (625)

Organotin Compounds (626)

Organovanadium Compounds (627)

D. Electrophoresis . . . . . . . . . . . . . . . 628

Organoiron Compounds (628)

Organolead Compounds (631)

Organophosphorus Compounds (631)

Organothallium Compounds (631)

CHAPTER 9 ORGANOMETALLIC COMPOUNDS IN THE ENVIRONMENT

A. Introductory . . . . . . . . . . . . . . . 632

B. Organometallic Compounds in Water . . . . . . . . . . 635

Organomercury Compounds (635)

Stability of Samples (635)

Natural Waters (637)

Potable Waters (649)

Sea and Coastal Waters (653)

Sewage and Trade Effluents (659)

Organogermanium Compounds (661)

Organotin Compounds (662)

Organolead Compounds (675)

Organoarsenic Compounds (685)

Organoantimony Compounds (699)

Organocopper Compounds (700)

Organosilicon Compounds (700)

C. Organometallic Compounds in Sediments . . . . . . . . . . 702

Organomercury Compounds (702)

Organotin Compounds (709)

Organolead Compounds (709)

Organoarsenic Compounds (713)

Organosilicon Compounds (714)

D. Organometallic Compounds in Plants . . . . . . . . . . . . 714 Organomercury Compounds (714)

Organotin Compounds (717)

Organolead Compounds (717)

Organomagnesium Compounds (718) 
E. Organometallic Compounds in Biological Materials . . . . . . 719 Organomercury Compounds in Fish (719)

Organomercury Compounds in Other Biological Materials (734)

Organotin Compounds (746)

Organolead Compounds (749)

Organoarsenic Compounds (754)

Organosilicon Compounds (758)

F. Organometallic Compounds in Air . . . . . . . . . . . . 758 Organomercury Compounds (758)

Organomanganese Compounds (763)

Organonickel Compounds (765)

Organotin Compounds (766)

Organolead Compounds (766)

Organosilicon Compounds (785)

REFERENCES . . . . . . . . . . . . . . . . . . 786

INDEX .......................... 875 